\title{
Regular Functions with Values in Ternary Number System on the Complex Clifford Analysis
}

\author{
Ji Eun Kim, Su Jin Lim, and Kwang Ho Shon \\ Department of Mathematics, Pusan National University, Busan 609-735, Republic of Korea \\ Correspondence should be addressed to Kwang Ho Shon; khshon@pusan.ac.kr
}

Received 21 October 2013; Accepted 5 December 2013

Academic Editor: Junesang Choi

Copyright (C) 2013 Ji Eun Kim et al. This is an open access article distributed under the Creative Commons Attribution License, which permits unrestricted use, distribution, and reproduction in any medium, provided the original work is properly cited.

We define a new modified basis $\hat{i}$ which is an association of two bases, $e_{1}$ and $e_{2}$. We give an expression of the form $z=x_{0}+\hat{i} \overline{z_{0}}$, where $x_{0}$ is a real number and $\overline{z_{0}}$ is a complex number on three-dimensional real skew field. And we research the properties of regular functions with values in ternary field and reduced quaternions by Clifford analysis.

\section{Introduction}

The noncommutative three-dimensional real field $\mathbb{R}^{3}$ of the hypercomplex numbers is called a ternary number system $\mathbb{T}$. The quaternions are represented by the form $z=\sum_{j=0}^{3} e_{j} x_{j}$, where $x_{j}(j=0, \ldots, 3)$ are real numbers on four dimensional real field $\mathbb{R}^{4}$. Fueter [1] has given a definition of quaternionic functions in $\mathbb{R}^{4}$ and Deavours [2] and Sudbery [3] have developed theories of quaternionic analysis. Naser [4] investigated some properties of hyperholomorphic functions and Koriyama et al. [5] researched properties of hyperholomorphic functions and holomorphic functions in quaternionic analysis. Nôno [6] obtained several results for regular functions which have a complex number form in quaternion analysis. Cho [7] researched some properties of Euler's formula and De moivre's formula for quaternions. Sangwine and Bihan [8] obtained some results for the quaternionic polar representation with a complex modulus and complex argument inspired by the cayley-dickson form. Fueter [9] obtained some properties of the three variables which are called the Fueter variables and researched the fact that structures lead to the set of all Fueter-regular functions in the general cases of Clifford analysis. By Brackx et al. [10], the theory of Fueter-regularity has been developed and generalized as quaternionic variables for theories of Clifford-valued regular functions.

Lim and Shon [11-13] researched the existence of hyperconjugate harmonic functions of octonion variables, properties of dual quaternion functions, and regularity of functions with values in a noncommutative subalgebra of complex matrix algebras.

We consider that ternary numbers are generated by a new basis $\hat{i}$ and give some properties of regular functions with values in $\mathbb{T}$. Also, we represent the corresponding Euler's formula for the form $z=x_{0}+\hat{i} \overline{z_{0}}$ and investigate calculating rules for regular functions in Clifford analysis. We research new representations of Fueter variables in reduced quaternions with $\hat{i}$ and some characteristics of regularity of functions on the Fueter variable system.

\section{Preliminaries}

The ternary number system $\mathbb{T}$ is a three dimensional noncommutative and associative real field by three bases $e_{0}, e_{1}$, and $e_{2}$ with the following rules:

$$
\begin{gathered}
e_{1}^{2}=e_{2}^{2}=-1, \quad e_{1} e_{2}=-e_{2} e_{1}, \\
\overline{e_{0}}=e_{0}, \quad \overline{e_{j}}=-e_{j} \quad(j=1,2) .
\end{gathered}
$$

The element $e_{0}$ is the identity of $\mathbb{T}$ and $e_{1}$ identifies the imaginary unit $\sqrt{-1}$ in the complex field. We consider an association of two bases $e_{1}$ and $e_{2}$ as follows:

$$
\widehat{i}:=\frac{a e_{1}+b e_{2}}{\sqrt{a^{2}+b^{2}}}=\alpha e_{1}+\beta e_{2} \quad \text { with } \hat{i}^{2}=-1,
$$

where $\alpha:=a / \sqrt{a^{2}+b^{2}}, \beta:=b / \sqrt{a^{2}+b^{2}}$, and $a, b$ are real numbers except both zeros. 
The number of the skew field $\mathbb{T}$ is

$$
\begin{aligned}
z & =x_{0}+e_{1} x_{1}+e_{2} x_{2} \\
& =x_{0}+\hat{i} \overline{z_{0}}
\end{aligned}
$$

where $x_{j}(j=0,1,2)$ are real variables, $\overline{z_{0}}=\gamma\left(x_{1}-x_{2} e_{1} e_{2}\right)$, and $\gamma:=\alpha+\beta e_{1} e_{2}$.

We define the ternary number system

$$
\mathbb{T}:=\left\{z \mid z=x_{0}+\hat{i} \overline{z_{0}}\right\} \text {. }
$$

The conjugate number $z^{*}$ of $z$ in $\mathbb{T}$ is given by the form:

$$
z^{*}=x_{0}-\hat{i} \overline{z_{0}} \text {. }
$$

And the norm $|z|$ of $z$ and the inverse $z^{-1}$ of $z$ are given by the following forms:

$$
\begin{aligned}
|z|=\sqrt{z z^{*}} & =\sqrt{x_{0}^{2}+\overline{z_{0}} z_{0}}=\sqrt{\sum_{j=0}^{2} x_{j}^{2}}, \\
z^{-1} & =\frac{z^{*}}{|z|^{2}} \quad(z \neq 0),
\end{aligned}
$$

where $z_{0}=\bar{\gamma}\left(x_{1}+x_{2} e_{1} e_{2}\right)$ and $\bar{\gamma}=\alpha-\beta e_{1} e_{2}$.

We define the addition and multiplication of two ternary numbers $z=x_{0}+\hat{i} \overline{z_{0}}$ and $w=y_{0}+\widehat{i} \overline{w_{0}}$ as follows:

$$
\begin{gathered}
z+w=\left(x_{0}+y_{0}\right)+\hat{i}\left(\overline{z_{0}}+\overline{w_{0}}\right), \\
z w=\left(x_{0} y_{0}-z_{0} \overline{w_{0}}\right)+\hat{i}\left(x_{0} \overline{w_{0}}+\overline{z_{0}} y_{0}\right) .
\end{gathered}
$$

Theorem 1. Let $z$ be an arbitrary number in $\mathbb{T}$. Then the corresponding Euler formula for $z$ is

$$
e^{z}=e^{x_{0}}\left(\cos \left|z_{0}\right|+\frac{z_{0}}{\left|z_{0}\right|} \hat{i} \sin \left|z_{0}\right|\right) .
$$

Moreover, taking logarithms of both sides, one obtains the equation as follows:

$$
\ln z=\ln |z|+\frac{z_{0}}{\left|z_{0}\right|} \hat{i} \cos ^{-1}\left(\frac{x_{0}}{|z|}\right) .
$$

Proof. For the number $z=x_{0}+\widehat{i} \overline{z_{0}}$ in $\mathbb{T}$, we get $\left|\widehat{i} \overline{z_{0}}\right|=\left|\overline{z_{0}}\right|=$ $\left|z_{0}\right|$ and $\left(\left(z_{0} /\left|z_{0}\right|\right) \hat{i}^{2}=-1\right.$. Then,

$$
\begin{aligned}
e^{z} & =e^{x_{0}+\hat{i} \bar{z}_{0}}=e^{x_{0}} e^{\left(\hat{i z_{0}} /\left|\hat{i} \bar{z}_{0}\right|\right)\left|\hat{i} \bar{z}_{0}\right|} \\
& =e^{x_{0}}\left(\cos \left|z_{0}\right|+\frac{z_{0}}{\left|z_{0}\right|} \hat{i} \sin \left|z_{0}\right|\right) .
\end{aligned}
$$

From

$$
\begin{array}{r}
z=|z|\left(\frac{x_{0}}{|z|}+\frac{z_{0}}{\left|z_{0}\right|} \hat{i} \frac{\left|z_{0}\right|}{|z|}\right) \\
=|z|\left\{\cos \left(\cos ^{-1}\left(\frac{x_{0}}{|z|}\right)\right)\right. \\
\left.+\frac{z_{0}}{\left|z_{0}\right|} \hat{i} \sin \left(\cos ^{-1}\left(\frac{x_{0}}{|z|}\right)\right)\right\},
\end{array}
$$

we have

$$
\ln z=\ln |z|+\frac{z_{0}}{\left|z_{0}\right|} \widehat{i} \cos ^{-1}\left(\frac{x_{0}}{|z|}\right) .
$$

We consider the following differential operators:

$$
\begin{aligned}
D & :=\frac{1}{2} \sum_{j=0}^{2} \overline{e_{j}} \frac{\partial}{\partial x_{j}}=\frac{1}{2}\left(\frac{\partial}{\partial x_{0}}-\hat{i} \frac{\partial}{\partial z_{0}}\right), \\
D^{*} & =\frac{1}{2} \sum_{j=0}^{2} e_{j} \frac{\partial}{\partial x_{j}}=\frac{1}{2}\left(\frac{\partial}{\partial x_{0}}+\hat{i} \frac{\partial}{\partial z_{0}}\right),
\end{aligned}
$$

where $\partial / \partial z_{0}=\gamma\left(\partial / \partial x_{1}-e_{1} e_{2}\left(\partial / \partial x_{2}\right)\right)$ and $\partial / \partial \overline{z_{0}}=\bar{\gamma}\left(\partial / \partial x_{1}+\right.$ $\left.e_{1} e_{2}\left(\partial / \partial x_{2}\right)\right)$. Then the Laplacian operator is

$$
4 \Delta:=D D^{*}=D^{*} D=\frac{\partial^{2}}{\partial x_{0}^{2}}+\frac{\partial^{2}}{\partial z_{0} \partial \overline{z_{0}}}=\sum_{j=0}^{2} \frac{\partial^{2}}{\partial x_{j}^{2}} .
$$

Let $\Omega$ be an open set in $\mathbb{R}^{3}$. The function $f(z)$ that is defined by the following form in $\Omega$ with values in $\mathbb{T}$ :

$$
f: \Omega \longrightarrow \mathbb{T}
$$

satisfies

$$
z=\left(x_{0}, \overline{z_{0}}\right) \in \Omega \longmapsto f(z)=u_{0}\left(x_{0}, \overline{z_{0}}\right)+\widehat{i f_{0}}\left(x_{0}, \overline{z_{0}}\right) \in \mathbb{T},
$$

where $u_{j}(j=0,1,2)$ are real-valued functions and

$$
f_{0}=\bar{\gamma}\left(u_{1}+u_{2} e_{1} e_{2}\right), \quad \overline{f_{0}}=\gamma\left(u_{1}-u_{2} e_{1} e_{2}\right)
$$

are complex-valued functions with values in $\mathbb{T}$.

Remark 2. The operators $D$ and $D^{*}$ act for the function $f(z)$ on $\mathbb{T}$ as follows:

$$
\begin{aligned}
& D f=\frac{1}{2}\left\{\left(\frac{\partial u_{0}}{\partial x_{0}}+\frac{\partial \overline{f_{0}}}{\partial \overline{z_{0}}}\right)+\hat{i}\left(\frac{\partial \overline{f_{0}}}{\partial x_{0}}-\frac{\partial u_{0}}{\partial z_{0}}\right)\right\}, \\
& D^{*} f=\frac{1}{2}\left\{\left(\frac{\partial u_{0}}{\partial x_{0}}-\frac{\partial \overline{f_{0}}}{\partial \overline{z_{0}}}\right)+\hat{i}\left(\frac{\partial \overline{f_{0}}}{\partial x_{0}}+\frac{\partial u_{0}}{\partial z_{0}}\right)\right\}, \\
& f D=\frac{1}{2}\left\{\left(\frac{\partial u_{0}}{\partial x_{0}}+\frac{\partial f_{0}}{\partial z_{0}}\right)+\hat{i}\left(\frac{\partial \overline{f_{0}}}{\partial x_{0}}-\frac{\partial u_{0}}{\partial z_{0}}\right)\right\}, \\
& f D^{*}=\frac{1}{2}\left\{\left(\frac{\partial u_{0}}{\partial x_{0}}-\frac{\partial f_{0}}{\partial z_{0}}\right)+\hat{i}\left(\frac{\partial \overline{f_{0}}}{\partial x_{0}}+\frac{\partial u_{0}}{\partial z_{0}}\right)\right\} .
\end{aligned}
$$

\section{Properties of Regular Functions with Values in $\mathbb{T}$}

Definition 3. Let $\Omega$ be an open set in $\mathbb{R}^{3}$. A function $f(z)=$ $u_{0}\left(x_{0}, \overline{z_{0}}\right)+\widehat{i f_{0}}\left(x_{0}, \overline{z_{0}}\right)$ is said to be $\mathrm{L}(\mathrm{R})$-regular in $\Omega$, if the following two conditions are satisfied:

(i) $u_{0}$ and $f_{0}$ are continuously differential functions on $\Omega$; 
(ii) $D^{*} f(z)=0\left(f(z) D^{*}=0\right)$ on $\Omega$.

Remark 4. The left equation (ii) of Definition 3 is equivalent to the following:

$$
\frac{\partial u_{0}}{\partial x_{0}}=\frac{\partial \overline{f_{0}}}{\partial \overline{z_{0}}}, \quad \frac{\partial \overline{f_{0}}}{\partial x_{0}}=-\frac{\partial u_{0}}{\partial z_{0}} .
$$

The equations in (19) are called the corresponding CauchyRiemann system for $f(z)$ in $\mathbb{T}$. The right equation (ii) of Definition 3 is equivalent to (19). When the function $f(z)=$ $u_{0}\left(x_{0}, \overline{z_{0}}\right)+\widehat{i f_{0}}\left(x_{0}, \overline{z_{0}}\right)$ is a L-regular function on $\Omega \subset \mathbb{R}^{3}$, simply we say that $f(z)$ is a regular function on $\Omega \subset \mathbb{R}^{3}$. In this case, we often say that $f(z)$ is a biregular function on $\Omega \subset \mathbb{R}^{3}$.

Remark 5. Let $\Omega$ be an open set in $\mathbb{R}^{3}$ and let $f(z)$ be a regular function on $\Omega$. Then we can replace the corresponding Cauchy-Riemann system in $\mathbb{R}^{3}$ as follows:

$$
\begin{array}{cc}
\frac{\partial u_{0}}{\partial x_{0}}=\frac{\partial u_{1}}{\partial x_{1}}+\frac{\partial u_{2}}{\partial x_{2}}, & \frac{\partial u_{1}}{\partial x_{2}}=\frac{\partial u_{2}}{\partial x_{1}}, \\
\frac{\partial u_{0}}{\partial x_{1}}=-\frac{\partial u_{1}}{\partial x_{0}}, & \frac{\partial u_{0}}{\partial x_{2}}=-\frac{\partial u_{2}}{\partial x_{0}}
\end{array}
$$

where $u_{j}(j=0,1,2)$ are real-valued functions.

Theorem 6. Let $\Omega$ be an open set in $\mathbb{R}^{3}$ and let $f$ be a regular function on $\Omega$. Then the derivative $f^{\prime}$ of $f$ defined by $D f$ is

$$
f^{\prime}=\frac{\partial f}{\partial x_{0}}=-\hat{i} \frac{\partial f}{\partial z_{0}}
$$

on $\Omega$.

Proof. By the definition of regular function with values in $\mathbb{T}$, we have

$$
\begin{aligned}
D f & =\frac{1}{2}\left\{\left(\frac{\partial u_{0}}{\partial x_{0}}+\frac{\partial \overline{f_{0}}}{\partial \overline{z_{0}}}\right)+\widehat{i}\left(\frac{\partial \overline{f_{0}}}{\partial x_{0}}-\frac{\partial u_{0}}{\partial z_{0}}\right)\right\} \\
& =\frac{\partial u_{0}}{\partial x_{0}}+\widehat{i} \frac{\partial \overline{f_{0}}}{\partial x_{0}}=\frac{\partial f}{\partial x_{0}}
\end{aligned}
$$

on $\Omega$. And

$$
D f=\frac{\partial \overline{f_{0}}}{\partial \overline{z_{0}}}-\hat{i} \frac{\partial u_{0}}{\partial z_{0}}=-\hat{i}\left(\frac{\partial}{\partial z_{0}} \widetilde{i f_{0}}+\frac{\partial u_{0}}{\partial z_{0}}\right)=-\hat{i} \frac{\partial f}{\partial z_{0}}
$$

on $\Omega$.

Theorem 7. Let $\Omega$ be an open set in $\mathbb{R}^{3}$ and let $f=u_{0}+\widehat{i f_{0}}$ be a function with values in $\mathbb{T}$. Suppose that $\partial f / \partial x_{0}$ and $\partial f / \partial z_{0}$ exist and are continuous on $\Omega$. If

$$
\frac{\partial f}{\partial x_{0}}=-\hat{i} \frac{\partial f}{\partial z_{0}}
$$

on $\Omega$, then $f$ is regular on $\Omega$.
Proof. Since $\partial f / \partial x_{0}=-\hat{i}\left(\partial f / \partial z_{0}\right)$, we have

$$
\frac{\partial f}{\partial x_{0}}=\frac{\partial u_{0}}{\partial x_{0}}+\widehat{i} \frac{\partial \overline{f_{0}}}{\partial x_{0}} .
$$

Hence, we have $D^{*} f=0$ and then $f$ is regular on $\Omega$.

Definition 8. Let $\Omega$ be an open set in $\mathbb{R}^{3}$. A function $f=u_{0}+$ $\widehat{i f_{0}}$ is said to be harmonic on $\Omega$ if all its components $u_{0}$ and $\overline{f_{0}}$ of $f$ are harmonic on $\Omega$.

Proposition 9. Let $\Omega$ be an open set in $\mathbb{R}^{3}$. If the function $f$ is regular on $\Omega$, then $f$ is harmonic on $\Omega$.

Proof. Since $f$ is regular function on $\Omega$, we have

$$
\begin{aligned}
D D^{*} \overline{f_{0}}=\frac{1}{4} & \left\{\left(\frac{\partial}{\partial x_{0}} \frac{\partial \overline{f_{0}}}{\partial x_{0}}+\frac{\partial}{\partial \overline{z_{0}}} \frac{\partial \overline{f_{0}}}{\partial z_{0}}\right)\right. \\
& \left.+\hat{i}\left(\frac{\partial}{\partial x_{0}} \frac{\partial \overline{f_{0}}}{\partial z_{0}}-\frac{\partial}{\partial z_{0}} \frac{\partial \overline{f_{0}}}{\partial x_{0}}\right)\right\}=0 .
\end{aligned}
$$

Similarly, we can prove that $D D^{*} u_{0}=0$. So, we obtain the result.

Proposition 10. Let $\Omega$ be an open set in $\mathbb{R}^{3}$ and let $f=u_{0}+\widetilde{i f_{0}}$ and $g=v_{0}+\hat{i} \overline{g_{0}}$ be regular functions on $\Omega$. Then the following properties hold:

(i) $f \alpha$ is regular on $\Omega$, if $\alpha$ is any ternary constant;

(ii) $\alpha f$ is not regular on $\Omega$, if $\alpha$ is any ternary constant;

(iii) $f \pm g$ is regular on $\Omega$;

(iv) $\mathrm{fg}$ is not regular on $\Omega$. Moreover, if $g$ is a real-valued function, then $\mathrm{fg}$ is regular on $\Omega$.

Proof. It is sufficient to show the second condition of Definition 3.

(i) Let $\alpha$ be a ternary constant with $\alpha=a_{0}+\hat{i} \overline{\alpha_{0}}$, where

$$
\alpha_{0}=\frac{c_{1} a_{1}+c_{2} a_{2}}{\sqrt{c_{1}^{2}+c_{2}^{2}}}+\frac{c_{2} a_{1}-c_{1} a_{2}}{\sqrt{c_{1}^{2}+c_{2}^{2}}} e_{1} e_{2}
$$

and $a_{0}, a_{1}, a_{2}, c_{1}$, and $c_{2}$ are real numbers. Then the equation

$$
\begin{aligned}
D^{*}(f \alpha)= & \frac{1}{2}\left(\frac{\partial}{\partial x_{0}}+\hat{i} \frac{\partial}{\partial z_{0}}\right) \\
& \times\left\{\left(u_{0} a_{0}-f_{0} \overline{\alpha_{0}}\right)+\hat{i}\left(u_{0} \overline{\alpha_{0}}+\overline{f_{0}} a_{0}\right)\right\} \\
= & \frac{1}{2}\left(\left(\frac{\partial u_{0}}{\partial x_{0}} a_{0}-\frac{\partial f_{0}}{\partial x_{0}} \overline{\alpha_{0}}-\frac{\partial u_{0}}{\partial \overline{z_{0}}} \overline{\alpha_{0}}-\frac{\partial \overline{f_{0}}}{\partial \overline{z_{0}}} a_{0}\right)\right. \\
& \left.+\hat{i}\left(\frac{\partial u_{0}}{\partial x_{0}} \overline{\alpha_{0}}+\frac{\partial \overline{f_{0}}}{\partial x_{0}} a_{0}+\frac{\partial u_{0}}{\partial z_{0}} a_{0}-\frac{\partial f_{0}}{\partial z_{0}} \overline{\alpha_{0}}\right)\right) \\
= & 0 .
\end{aligned}
$$

Hence, $f \alpha$ is regular on $\Omega$. 
(ii) Since

$$
\begin{aligned}
D^{*}(\alpha f)= & \frac{1}{2}\left(\frac{\partial}{\partial x_{0}}+\hat{i} \frac{\partial}{\partial z_{0}}\right) \\
& \times\left\{\left(a_{0} u_{0}-\alpha_{0} \overline{f_{0}}\right)+\hat{i}\left(a_{0} \overline{f_{0}}+\overline{\alpha_{0}} u_{0}\right)\right\} \\
= & \frac{1}{2}\left(\left(a_{0} \frac{\partial u_{0}}{\partial x_{0}}-\alpha_{0} \frac{\partial \overline{f_{0}}}{\partial x_{0}}-\overline{\alpha_{0}} \frac{\partial f_{0}}{\partial x_{0}}-\frac{\partial u_{0}}{\partial z_{0}} \alpha_{0}\right)\right. \\
& \left.+\widehat{i}\left(a_{0} \frac{\partial \overline{f_{0}}}{\partial x_{0}}+\overline{\alpha_{0}} \frac{\partial u_{0}}{\partial x_{0}}+a_{0} \frac{\partial u_{0}}{\partial z_{0}}-\alpha_{0} \frac{\partial \overline{f_{0}}}{\partial z_{0}}\right)\right)
\end{aligned}
$$

is not zero, $\alpha f$ is not always regular on $\Omega$.

(iii) Since

$$
\begin{aligned}
D^{*}(f \pm g)= & \frac{1}{2}\left(\frac{\partial}{\partial x_{0}}+\hat{i} \frac{\partial}{\partial z_{0}}\right)\left\{\left(u_{0} \pm v_{0}\right)+\hat{i}\left(\overline{f_{0}} \pm \overline{g_{0}}\right)\right\} \\
= & \frac{1}{2}\left(\left(\frac{\partial u_{0}}{\partial x_{0}} \pm \frac{\partial v_{0}}{\partial x_{0}}-\frac{\partial \overline{f_{0}}}{\partial \overline{z_{0}}} \mp \frac{\partial \overline{g_{0}}}{\partial \overline{z_{0}}}\right)\right. \\
& \left.+\hat{i}\left(\frac{\partial u_{0}}{\partial z_{0}} \pm \frac{\partial v_{0}}{\partial z_{0}}+\frac{\partial \overline{f_{0}}}{\partial x_{0}} a_{0} \pm \frac{\partial \overline{g_{0}}}{\partial x_{0}}\right)\right)=0,
\end{aligned}
$$

$f \pm g$ is regular on $\Omega$.

(iv) Since

$$
\begin{aligned}
& D^{*}(f g)=\frac{1}{2}\left(\frac{\partial}{\partial x_{0}}+\hat{i} \frac{\partial}{\partial z_{0}}\right) \\
& \times\left\{\left(u_{0} v_{0}-f_{0} \overline{g_{0}}\right)+\hat{i}\left(u_{0} \overline{g_{0}}+\overline{f_{0}} v_{0}\right)\right\} \\
& =\frac{1}{2}\left(\left(\frac{\partial u_{0}}{\partial x_{0}}-\frac{\partial \overline{f_{0}}}{\partial \overline{z_{0}}}\right) v_{0}+u_{0}\left(\frac{\partial v_{0}}{\partial x_{0}}-\frac{\partial \overline{g_{0}}}{\partial \overline{z_{0}}}\right)\right. \\
& -\left(\frac{\partial f_{0}}{\partial x_{0}}+\frac{\partial u_{0}}{\partial \overline{z_{0}}}\right) \overline{g_{0}}-\left(f_{0} \frac{\partial \overline{g_{0}}}{\partial x_{0}}+\overline{f_{0}} \frac{\partial v_{0}}{\partial \overline{z_{0}}}\right) \\
& +\hat{i}\left\{\left(\frac{\partial u_{0}}{\partial x_{0}}-\frac{\partial f_{0}}{\partial z_{0}}\right) \overline{g_{0}}+u_{0}\left(\frac{\partial \overline{g_{0}}}{\partial x_{0}}+\frac{\partial v_{0}}{\partial z_{0}}\right)\right. \\
& +\left(\frac{\partial \overline{f_{0}}}{\partial x_{0}}+\frac{\partial u_{0}}{\partial z_{0}}\right) v_{0} \\
& \left.\left.+\left(\overline{f_{0}} \frac{\partial v_{0}}{\partial x_{0}}-f_{0} \frac{\partial \overline{g_{0}}}{\partial z_{0}}\right)\right\}\right) \\
& =\frac{1}{2}\left(-\left(f_{0} \frac{\partial \overline{g_{0}}}{\partial x_{0}}+\overline{f_{0}} \frac{\partial v_{0}}{\partial \overline{z_{0}}}\right)+\hat{i}\left(\overline{f_{0}} \frac{\partial v_{0}}{\partial x_{0}}-f_{0} \frac{\partial \overline{g_{0}}}{\partial z_{0}}\right)\right)
\end{aligned}
$$

is not zero, $f g$ is not always regular on $\Omega$.
Theorem 11. Let $\Omega$ be an open set in $\mathbb{R}^{3}$ and let $f$ and $g$ be regular functions on $\Omega$. Then we have the following equations:

$$
\begin{aligned}
2 D^{*}(f g) & =\left(D^{*} f\right) g+f \frac{\partial g}{\partial x_{0}}+\widehat{i}\left(u_{0} \frac{\partial g}{\partial z_{0}}+\widetilde{i f_{0}} \frac{\partial g}{\partial \overline{z_{0}}}\right) . \\
2 D(f g) & =(D f) g+f \frac{\partial g}{\partial x_{0}}-\hat{i}\left(u_{0} \frac{\partial g}{\partial z_{0}}+\widetilde{i f_{0}} \frac{\partial g}{\partial \overline{z_{0}}}\right) .
\end{aligned}
$$

Proof. From the proof of Proposition 10, we have the following equations:

$$
\begin{aligned}
2 D^{*}(f g)= & \left\{\left(\frac{\partial u_{0}}{\partial x_{0}}-\frac{\partial \overline{f_{0}}}{\partial \overline{z_{0}}}\right)+\hat{i}\left(\frac{\partial \overline{f_{0}}}{\partial x_{0}}+\frac{\partial u_{0}}{\partial z_{0}}\right)\right\}\left(v_{0}+\hat{i} \overline{g_{0}}\right) \\
& -\left(f_{0} \frac{\partial \overline{g_{0}}}{\partial x_{0}}+\overline{f_{0}} \frac{\partial v_{0}}{\partial \overline{z_{0}}}\right)+\hat{i}\left(\overline{f_{0}} \frac{\partial v_{0}}{\partial x_{0}}-f_{0} \frac{\partial \overline{g_{0}}}{\partial z_{0}}\right) \\
= & \left\{\left(\frac{\partial u_{0}}{\partial x_{0}}-\frac{\partial \overline{f_{0}}}{\partial \overline{z_{0}}}\right)+\hat{i}\left(\frac{\partial \overline{f_{0}}}{\partial x_{0}}+\frac{\partial u_{0}}{\partial z_{0}}\right)\right\}\left(v_{0}+\hat{i \overline{g_{0}}}\right) \\
& +u_{0}\left(\frac{\partial v_{0}}{\partial x_{0}}-\frac{\partial \overline{g_{0}}}{\partial \overline{z_{0}}}\right)-\left(f_{0} \frac{\partial \overline{g_{0}}}{\partial x_{0}}+\overline{f_{0}} \frac{\partial v_{0}}{\partial \overline{z_{0}}}\right) \\
& +\hat{i} u_{0}\left(\frac{\partial \overline{g_{0}}}{\partial x_{0}}+\frac{\partial v_{0}}{\partial z_{0}}\right)+\hat{i}\left(\overline{f_{0}} \frac{\partial v_{0}}{\partial x_{0}}-f_{0} \frac{\partial \overline{g_{0}}}{\partial z_{0}}\right) \\
= & \left(D^{*} f\right) g+f \frac{\partial g}{\partial x_{0}}+\hat{i}\left(u_{0} \frac{\partial g}{\partial z_{0}}+\widehat{i f_{0}} \frac{\partial g}{\partial \overline{z_{0}}}\right) .
\end{aligned}
$$

Similarly, we can prove (33)

We let

$$
k=e_{1} e_{2} \frac{1}{2} d z_{0} \wedge d \overline{z_{0}}+e_{2} \alpha d x_{0} \wedge d \overline{z_{0}}-e_{1} \beta d x_{0} \wedge d \overline{z_{0}} .
$$

Theorem 12. Let $\Omega$ be an open set in $\mathbb{R}^{3}$ and $U$ be any domain in $\Omega$ with smooth boundarybU such that $U \subset \Omega$. If $f=u_{0}+\widetilde{i f_{0}}$ is a regular function on $\Omega$, then

$$
\int_{b U} k f=0
$$

where $k f$ is the ternary product of the form $k$ on the function $f(z)$.

Proof. Since the function $f=u_{0}+e_{1} \alpha \overline{f_{0}}+e_{2} \beta \overline{f_{0}}$ exists, we have

$$
\begin{aligned}
k f= & \left(e_{1} e_{2} \frac{1}{2} u_{0}-e_{2} \frac{1}{2} \alpha \overline{f_{0}}+e_{1} \frac{1}{2} \beta \overline{f_{0}}\right) d z_{0} \wedge d \overline{z_{0}} \\
& +\left(e_{2} \alpha u_{0}-e_{1} \beta u_{0}\right) d x_{0} \wedge d \overline{z_{0}} \\
& +\left(-e_{1} e_{2} \alpha^{2} \overline{f_{0}}-e_{1} e_{2} \beta^{2} \overline{f_{0}}\right) d x_{0} \wedge d z_{0} .
\end{aligned}
$$


Then

$$
\begin{aligned}
d(k f)= & e_{1} e_{2}\left(\frac{\partial u_{0}}{\partial x_{0}}-\alpha^{2} \frac{\partial \overline{f_{0}}}{\partial \overline{z_{0}}}-\beta^{2} \frac{\partial \overline{f_{0}}}{\partial \overline{z_{0}}}\right) d V \\
& +e_{2}\left(-\alpha \frac{\partial \overline{f_{0}}}{\partial x_{0}}-\alpha \frac{\partial u_{0}}{\partial z_{0}}\right) d V \\
& +e_{1}\left(\beta \frac{\partial \overline{f_{0}}}{\partial x_{0}}+\beta \frac{\partial u_{0}}{\partial z_{0}}\right) d V \\
& +\left(-\alpha \beta \frac{\partial \overline{f_{0}}}{\partial \overline{z_{0}}}+\alpha \beta \frac{\partial \overline{f_{0}}}{\partial \overline{z_{0}}}\right) d V \\
= & \left\{e_{1} e_{2}\left(\frac{\partial u_{0}}{\partial x_{0}}-\frac{\partial \overline{f_{0}}}{\partial \overline{z_{0}}}\right)-e_{2} \alpha\left(\frac{\partial \overline{f_{0}}}{\partial x_{0}}+\frac{\partial u_{0}}{\partial z_{0}}\right)\right. \\
& \left.+e_{1} \beta\left(\frac{\partial \overline{f_{0}}}{\partial x_{0}}+\frac{\partial u_{0}}{\partial z_{0}}\right)\right\} d V,
\end{aligned}
$$

where $d V=d x_{0} \wedge d z_{0} \wedge d \overline{z_{0}}$ in $U$, and by the corresponding Cauchy-Riemann system for $f(z)$ in $\mathbb{T}, d(k f)=0$. By Stokes theorem, we obtain the result.

Remark 13. Since

$$
\left(\hat{\bar{i} \bar{z}_{0}}\right)^{k}= \begin{cases}(-1)^{k / 2}\left(\left|z_{0}\right|\right)^{k}, & k: \text { even } \\ (-1)^{[k / 2]} \hat{i}\left(\left|z_{0}\right|\right)^{k-1} \overline{z_{0}}, & k: \text { odd, }\end{cases}
$$

we have

$$
z^{n}=\sum_{k=0}^{n} \alpha(k) x_{0}^{n-k}\left|z_{0}\right|^{[k / 2]} \bar{z}_{0}^{\delta_{k}}
$$

where

$$
\begin{gathered}
\alpha(k)=\left\{\begin{array}{l}
\left(\begin{array}{l}
n \\
k
\end{array}\right)(-1)^{k / 2}, \quad k: \text { even } \\
\left(\begin{array}{l}
n \\
k
\end{array}\right)(-1)^{[k / 2]} \hat{i}, \quad k: \text { odd },
\end{array}\right. \\
\delta_{k}= \begin{cases}0, & k: \text { even } \\
1, & k: \text { odd } .\end{cases}
\end{gathered}
$$

And $[k / 2]$ is the greatest integer that is less than or equal to $k / 2$.

Theorem 14. Let $f$ be a homogeneous polynomial of degree $n$ with respect to the variables $x_{0}$ and $\overline{z_{0}}$. If $f$ is regular on $\Omega$, then

$$
\begin{gathered}
f(z)=\frac{1}{n !} \frac{\partial^{n} f(z)}{\partial x_{0}^{n}} z^{n}, \\
f(z)=(-\hat{i})^{n} \frac{1}{n !} \frac{\partial^{n} f(z)}{\partial z_{0}^{n-r} \partial{\overline{z_{0}}}^{r}} z^{n},
\end{gathered}
$$

where $r$ is a nonnegative integer.
Proof. Since $f(z)$ is a homogeneous polynomial, then

$$
f(z)=\frac{1}{n} \frac{\partial f(z)}{\partial x_{0}} z .
$$

Also, since $\partial f(z) / \partial x_{0}$ is a homogeneous polynomial of degree $n-1$, we have

$$
\frac{\partial f(z)}{\partial x_{0}}=\frac{1}{n-1} \frac{\partial^{2} f(z)}{\partial x_{0}^{2}} z
$$

Then we have

$$
f(z)=\frac{1}{n(n-1)} \frac{\partial^{2} f(z)}{\partial x_{0}^{2}} z^{2} .
$$

Continuing this process, we can get the result (42). Similarly, we obtain the result (43).

\section{Properties of Regular Functions with Values in $\mathbb{T}(\mathbb{C})$}

We define the number system

$$
\mathbb{T}(\mathbb{C})=\left\{z \mid z=\hat{i} \gamma\left(z_{1}-e_{1} e_{2} z_{2}\right)\right\},
$$

where $z_{1}=x_{1}-(1 / 2) e_{1} x_{0}$ and $z_{2}=x_{2}-(1 / 2) e_{2} x_{0}$.

The non-commutative multiplication of two numbers $z=$ $\hat{i} \gamma\left(z_{1}-e_{1} e_{2} z_{2}\right)$ and $w=\hat{i} \gamma\left(w_{1}-e_{1} e_{2} w_{2}\right)$ is defined by

$$
\begin{aligned}
& z w=-\left\{\left(z_{1} w_{1}+z_{2} w_{2}\right)+e_{1} e_{2}\left(\overline{z_{2}} w_{1}-\overline{z_{1}} w_{2}\right)\right\}, \\
& w z=-\left\{\left(w_{1} z_{1}+w_{2} z_{2}\right)+e_{1} e_{2}\left(\overline{w_{2}} z_{1}-\overline{w_{1}} z_{2}\right)\right\} .
\end{aligned}
$$

The conjugate number $z^{*}$ of $z$ in $\mathbb{T}(\mathbb{C})$ is given by the following:

$$
z^{*}=-\hat{i \gamma}\left(\overline{z_{1}}-e_{1} e_{2} \overline{z_{2}}\right) .
$$

And the norm $|z|$ of $z$ and the inverse $z^{-1}$ of $z$ are given by the following forms:

$$
\begin{aligned}
|z| & =\sqrt{z z^{*}}=\sqrt{z^{*} z} \\
& =\sqrt{\left(z_{1} \overline{z_{1}}+z_{2} \overline{z_{2}}\right)+e_{1} e_{2}\left(\overline{z_{2} z_{1}}-\overline{z_{1} z_{2}}\right)} \\
& =\sqrt{\sum_{j=0}^{2} x_{j}^{2}}, \\
z^{-1} & =\frac{z^{*}}{|z|^{2}} \quad(z \neq 0) .
\end{aligned}
$$

We consider the following differential operators:

$$
D=-\frac{1}{2} \hat{i} \gamma\left(D_{z_{1}}-e_{1} e_{2} D_{z_{2}}\right), \quad D^{*}=\frac{1}{2} \hat{i} \gamma\left(D_{\overline{z_{1}}}-e_{1} e_{2} D_{\overline{z_{2}}}\right),
$$

where

$$
D_{z_{1}}=\frac{1}{2} e_{1} \frac{\partial}{\partial x_{0}}+\frac{\partial}{\partial x_{1}}, \quad D_{z_{2}}=\frac{1}{2} e_{2} \frac{\partial}{\partial x_{0}}+\frac{\partial}{\partial x_{2}} .
$$


Then the Laplacian operator is

$$
4 \Delta:=D D^{*}=D^{*} D=\sum_{j=0}^{2} \frac{\partial^{2}}{\partial x_{j}^{2}} .
$$

Let $G$ be an open set in $\mathbb{C}^{2}$. The function $f(z)$ that is defined by the following form in $G$ with values in $\mathbb{T}(\mathbb{C})$ :

$$
f: G \rightarrow \mathbb{T}(\mathbb{C})
$$

satisfies

$$
\begin{aligned}
z & =\left(z_{1}, z_{2}\right) \in G \longmapsto f(z)=f\left(z_{1}, z_{2}\right) \\
& =\widehat{i} \gamma\left(f_{1}\left(z_{1}, z_{2}\right)-e_{1} e_{2} f_{2}\left(z_{1}, z_{2}\right)\right),
\end{aligned}
$$

where $f_{1}=u_{1}-(1 / 2) e_{1} u_{0}$ and $f_{2}=u_{2}-(1 / 2) e_{2} u_{0}$ are complex-valued functions with values in $\mathbb{T}(\mathbb{C})$ and $u_{j}(j=$ $0,1,2)$ are real-valued functions.

Remark 15. The operators $D$ and $D^{*}$ act for a function $f(z)$ on $\mathbb{T}(\mathbb{C})$ as follows:

$$
\begin{aligned}
& D f=-\hat{i}^{2}\left\{\left(D_{z_{1}} f_{1}+D_{z_{2}} f_{2}\right)+e_{1} e_{2}\left(D_{\overline{z_{2}}} f_{1}-D_{\overline{z_{1}}} f_{2}\right)\right\}, \\
& D^{*} f=\hat{i}^{2}\left\{\left(D_{\overline{z_{1}}} f_{1}+D_{\overline{z_{2}}} f_{2}\right)+e_{1} e_{2}\left(D_{z_{2}} f_{1}-D_{z_{1}} f_{2}\right)\right\} .
\end{aligned}
$$

We define a commutative multiplication of two numbers $z=\widehat{i} \gamma\left(z_{1}-e_{1} e_{2} z_{2}\right)$ and $w=\widehat{i} \gamma\left(w_{1}-e_{1} e_{2} w_{2}\right)$ by

$$
\begin{gathered}
z \odot w=w \odot z=\frac{1}{2}(z w+w z) \\
=\frac{1}{2} \widehat{i}^{2}\left\{\left(z_{1} w_{1}+z_{2} w_{2}+w_{1} z_{1}+w_{2} z_{2}\right)\right. \\
+e_{1} e_{2}\left(\overline{z_{2}} w_{1}-\overline{z_{1}} w_{2}\right. \\
\left.\left.+\overline{w_{2}} z_{1}-\overline{w_{1}} z_{2}\right)\right\} .
\end{gathered}
$$

Remark 16. The operators $D$ and $D^{*}$ act for a function $f(z)$ on $\mathbb{T}(\mathbb{C})$ as follows:

$$
\begin{aligned}
D \odot f= & \frac{1}{2}(D f+f D) \\
= & \left\{\left(D_{z_{1}} f_{1}+D_{z_{2}} f_{2}\right)\right. \\
& +\frac{1}{2} e_{1} e_{2}\left(D_{\overline{z_{2}}} f_{1}-D_{\overline{z_{1}}} f_{2}\right. \\
& \left.\left.+\overline{f_{2}} D_{z_{1}}-\overline{f_{1}} D_{z_{2}}\right)\right\} \\
= & \left\{\left(D_{z_{1}} f_{1}+D_{z_{2}} f_{2}+\frac{1}{2} \frac{\partial u_{0}}{\partial x_{0}}\right)\right. \\
& +\frac{1}{2} e_{1} e_{2}\left(D_{\overline{z_{2}}} f_{1}-D_{\overline{z_{1}}} f_{2}\right. \\
& \left.\left.+D_{z_{1}} \overline{f_{2}}-D_{z_{2}} \overline{f_{1}}\right)\right\},
\end{aligned}
$$

$$
\begin{aligned}
D^{*} \odot f= & \frac{1}{2}\left(D^{*} f+f D^{*}\right) \\
=- & \left\{\left(D_{\overline{z_{1}}} f_{1}+D_{\overline{z_{2}}} f_{2}\right)\right. \\
& +\frac{1}{2} e_{1} e_{2}\left(D_{z_{2}} f_{1}-D_{z_{1}} f_{2}\right. \\
& \left.\left.+\overline{f_{2}} D_{\overline{z_{1}}}-\overline{f_{1}} D_{\overline{z_{2}}}\right)\right\} \\
=- & \left\{\left(D_{\overline{z_{1}}} f_{1}+D_{\overline{z_{2}}} f_{2}-\frac{1}{2} \frac{\partial u_{0}}{\partial x_{0}}\right)\right. \\
& +\frac{1}{2} e_{1} e_{2}\left(D_{z_{2}} f_{1}-D_{z_{1}} f_{2}\right. \\
& \left.\left.+D_{\overline{z_{1}}} \overline{f_{2}}-D_{\overline{z_{2}}} \overline{f_{1}}\right)\right\} .
\end{aligned}
$$

Definition 17. Let $G$ be a domain in $\mathbb{C}^{2}$. A function $f=$ $\hat{i} \gamma\left(f_{1}-e_{1} e_{2} f_{2}\right)$ is said to be dot-regular in $G$ if the following two conditions are satisfied:

(i) $f_{1}$ and $f_{2}$ are differential functions in $G$,

(ii) $D^{*} \odot f=0$ in $G$.

Remark 18. The above equation (ii) of Definition 17 is equivalent as follows:

$$
\begin{gathered}
D_{\overline{z_{1}}} f_{1}+D_{\overline{z_{2}}} f_{2}=\frac{1}{2} \frac{\partial u_{0}}{\partial x_{0}}, \\
D_{z_{2}} f_{1}-D_{z_{1}} f_{2}=D_{\overline{z_{2}}} \overline{f_{1}}-D_{\overline{z_{1}}} \overline{f_{2}} .
\end{gathered}
$$

Theorem 19. Let $G$ be an open set in $\mathbb{C}^{2}$ and let $f$ be a dotregular function on $G$. Then the derivative $f^{\prime}$ of $f$ defined by $D \odot f$ is

$$
\begin{aligned}
f^{\prime} & =2 \hat{i} \gamma\left(D_{\overline{z_{1}}}-D_{z_{1}}\right) f=2 e_{1}\left(D_{\overline{z_{1}}}-D_{z_{1}}\right) f, \\
f^{\prime} & =-2 \widehat{i \gamma}\left(D_{z_{2}}-D_{\overline{z_{2}}}\right) f=2 e_{2}\left(D_{\overline{z_{2}}}-D_{z_{2}}\right) f .
\end{aligned}
$$

Proof. By the definition of a dot-regular function with values in $\mathbb{T}(\mathbb{C})$, we have

$$
\begin{aligned}
D \odot f= & \left(D_{\overline{z_{1}}} f_{1}+D_{\overline{z_{2}}} f_{2}+e_{1} \frac{\partial u_{1}}{\partial x_{0}}+e_{2} \frac{\partial u_{2}}{\partial x_{0}}+\frac{3}{2} \frac{\partial u_{0}}{\partial x_{0}}\right) \\
+ & \frac{1}{2} e_{1} e_{2}\left(D_{z_{2}} f_{1}-D_{z_{1}} f_{2}+D_{\overline{z_{1}}} \overline{f_{2}}\right. \\
& \left.-D_{\overline{z_{2}}} \overline{f_{1}}-2 e_{2} \frac{\partial u_{1}}{\partial x_{0}}+2 e_{1} \frac{\partial u_{2}}{\partial x_{0}}\right) \\
= & 2 \widehat{i} \gamma\left(D_{\overline{z_{1}}}-D_{z_{1}}\right) f
\end{aligned}
$$

on $G$. And, similarly, we have

$$
D \odot f=-2 \widehat{i} \gamma\left(D_{z_{2}}-D_{\overline{z_{2}}}\right) f
$$

on $G$. 


\section{Acknowledgment}

Kwang Ho Shon was supported by the Research Fund Program of Research Institute for Basic Sciences, Pusan National University, Korea, 2013, Project no. RIBS-PNU-2013-101.

\section{References}

[1] R. Fueter, "Die funktionentheorie der differentialgleichungen $\Delta u=0$ und $\Delta \Delta u=0$ mit vier reellen variablen," Commentarii Mathematici Helvetici, vol. 7, no. 1, pp. 307-330, 1935.

[2] C. A. Deavours, “The quaternion calculus," The American Mathematical Monthly, vol. 80, pp. 995-1008, 1973.

[3] A. Sudbery, "Quaternionic analysis," Mathematical Proceedings of the Cambridge Philosophical Society, vol. 85, no. 2, pp. 199224, 1979.

[4] M. Naser, "Hyperholomorphic functions," Silberian Mathematical Journal, vol. 12, pp. 959-968, 1971.

[5] H. Koriyama, H. Mae, and K. Nôno, "Hyperholomorphic functions and holomorphic functions in quaternionic analysis," Bulletin of Fukuoka University of Education, vol. 60, pp. 1-9, 2011.

[6] K. Nôno, "Hyperholomorphic functions of a quaternion variable," Bulletin of Fukuoka University of Education, vol. 32, pp. 21-37, 1983.

[7] E. Cho, "De moivre's formula for quaternions," Applied Mathematics Letters, vol. 11, no. 6, pp. 33-35, 1998.

[8] S. J. Sangwine and N. L. Bihan, "Quaternion polar representation with a complex modulus and complex argument inspired by the cayley-dickson form," Advances in Applied Clifford Algebras, vol. 20, no. 1, pp. 111-120, 2010.

[9] R. Fueter, "Die theorie der regulären funktionen einer quaternionenvariablen," in Comptès Rendus du Congrès International des Mathèmaticiens, Oslo 1936, vol. 1, pp. 75-91, 1935.

[10] F. Brackx, R. Delanghe, and F. Sommen, Clifford Analysis, vol. 76 of Research Notes in Mathematics, 1982.

[11] S. J. Lim and K. H. Shon, "Hyperholomorphic fucntions and hyperconjugate harmonic functions of octonion variables," Journal of Inequalities and Applications, vol. 77, pp. 1-8, 2013.

[12] S. J. Lim and K. H. Shon, "Dual quaternion functions and its applications," Journal of Applied Mathematics, vol. 2013, Article ID 583813, 6 pages, 2013.

[13] S. J. Lim and K. H. Shon, "Regularity of functions with values in a non-commutativealgebra of complex matrix algebras," Preprint. 


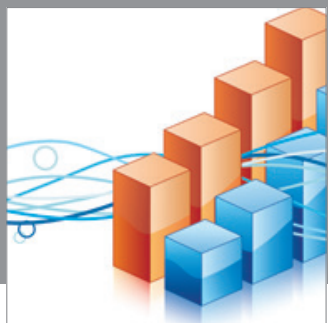

Advances in

Operations Research

mansans

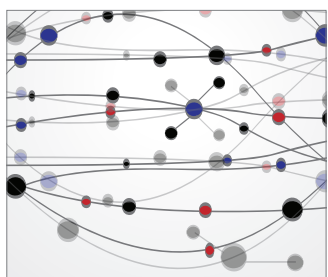

The Scientific World Journal
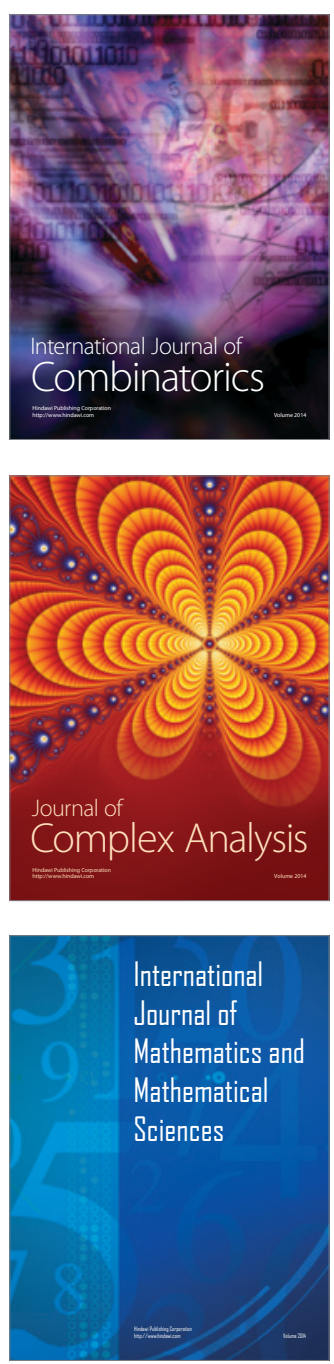
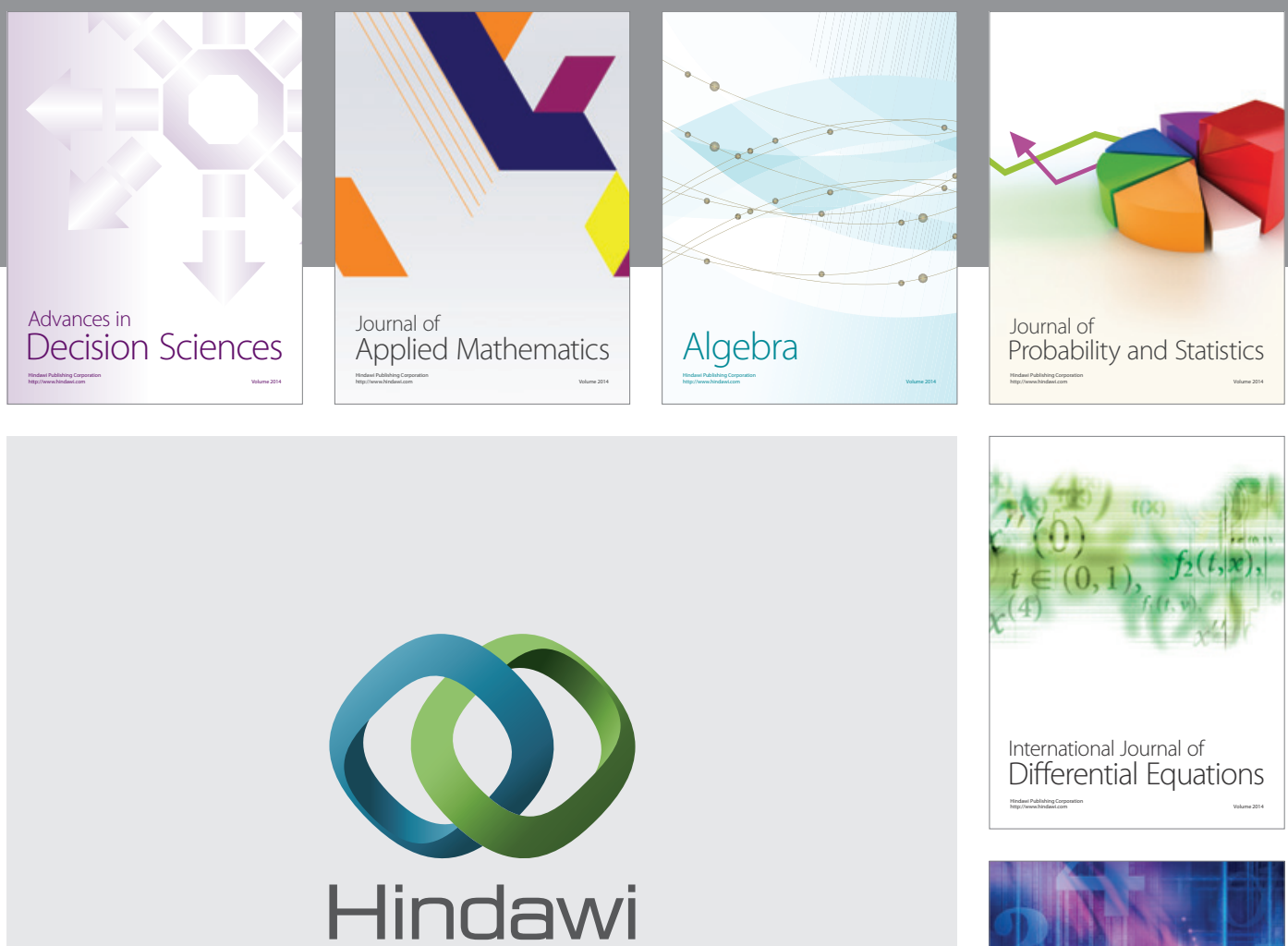

Submit your manuscripts at http://www.hindawi.com
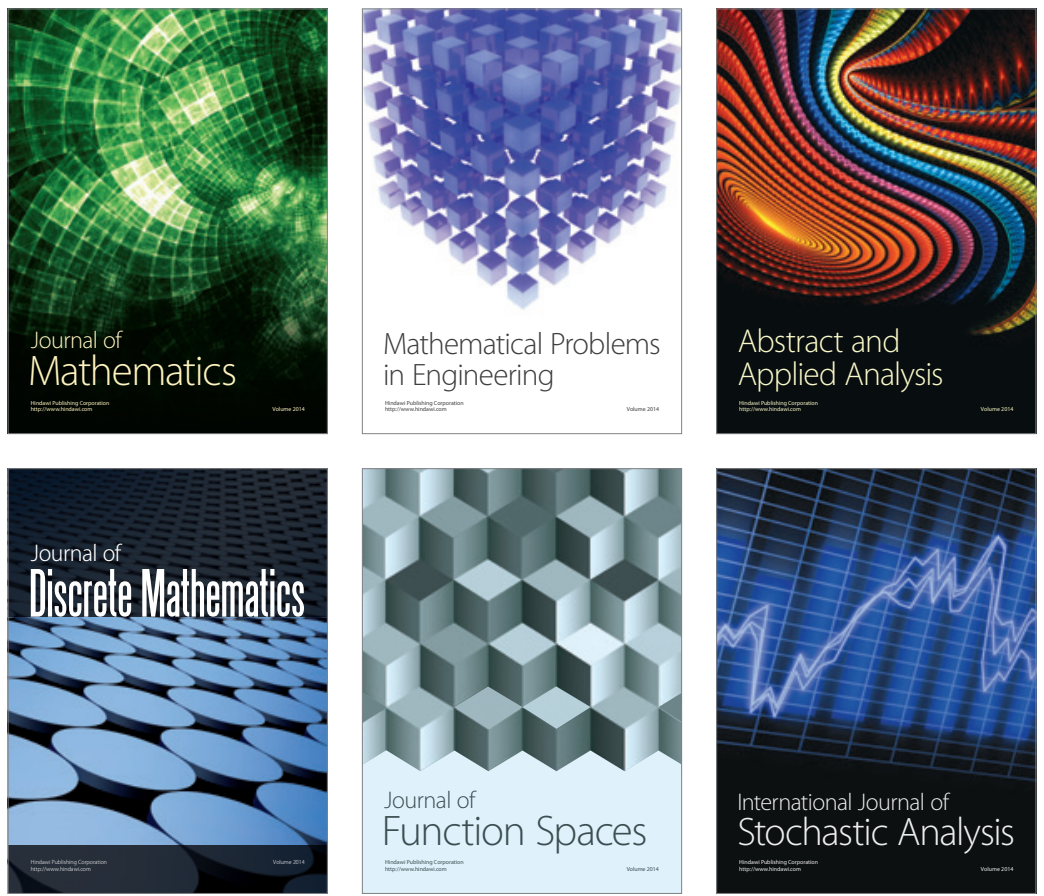

Journal of

Function Spaces

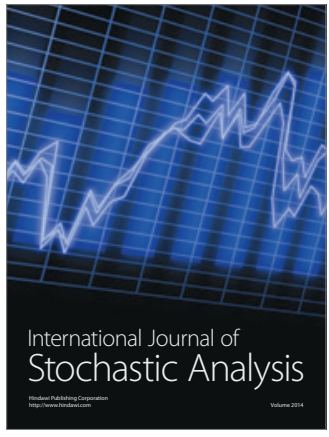

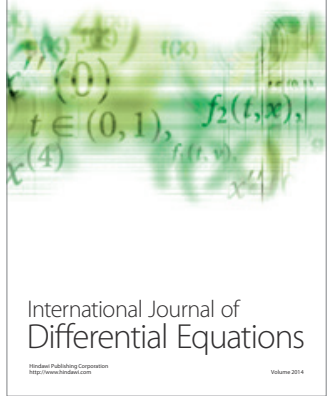
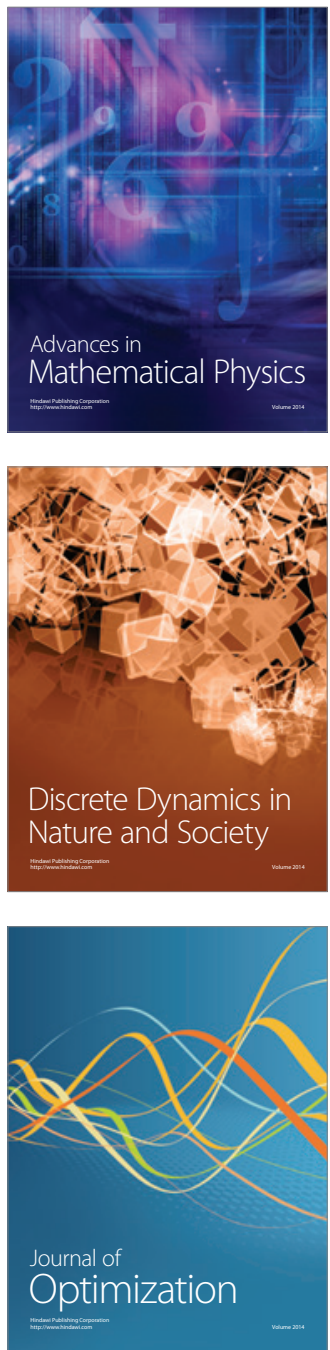